\title{
Gua Sha, a press-stroke treatment of the skin, boosts the immune response to intradermal vaccination
}

\author{
Tingting Chen ${ }^{1}$, Ninghua Liu ${ }^{1}$, Jinxuan Liu ${ }^{1}$, Xiaoying Zhang ${ }^{1}$, Zhen Huang ${ }^{1}$, Yuhui Zang ${ }^{1}$, Jiangning \\ Chen ${ }^{1}$, Lei Dong ${ }^{1}$, Junfeng Zhang ${ }^{1,2}$, Zhi Ding ${ }^{\text {Corresp. }{ }^{1,2}}$ \\ ${ }^{1}$ School of Life Sciences, Nanjing University, State Key Laboratory of Pharmaceutical Biotechnology, Nanjing, Jiangsu, China \\ 2 Nanjing University, Collaborative Innovation Center of Chemistry for Life Sciences, Nanjing, Jiangsu, China \\ Corresponding Author: Zhi Ding \\ Email address: dingzhi@nju.edu.cn
}

Objective: The skin is an important immunological barrier of the body as well as an optimal route for vaccine administration. Gua Sha, which involves press-stroke treatment of the skin, is an effective folk therapy, widely accepted in East Asia, for various symptoms; however, the mechanisms underlying its therapeutic effects have not been clarified. We investigated the influence of Gua Sha on the immunological features of the skin.

Methods: Gua Sha was performed on BALB/C mice and the effects were evaluated using anatomical, histological, and cytometric methods as well as cytokine determination locally and systemically. The effect on intradermal vaccination was assessed with antigen-specific subtype antibody responses.

Results: Blood vessel expansion, erythrocyte extravasation, and increased ratios of immune active cells were observed in the skin tissue following the treatment. Pro-inflammatory cytokines were up-regulated, and immunosuppressive cytokines, down-regulated, in the treated and untreated skin and systemic circulation; no obvious variations were detected in case of anti-inflammatory cytokines. Interestingly, intradermal delivery of a model vaccine following Gua Sha induced about three-fold higher IgG titers with a more Th1-biased antibody subtype profile.

Conclusion: Gua Sha treatment can up-regulate the innate and adaptive immune functions of the skin and boost the response against intradermal antigens. Thus, Gua Sha may serve as a safe, inexpensive, and independent physical adjuvant for intradermal vaccination. 
1 Gua Sha, a press-stroke treatment of the skin, boosts the

\section{immune response to intradermal vaccination}

3 Tingting Chen ${ }^{1 \uparrow}$, Ninghua Liu ${ }^{1 \uparrow}$, Jinxuan Liu ${ }^{1}$, Xiaoying Zhang ${ }^{1}$, Zhen Huang, Ph.D. ${ }^{1}$, Yuhui

4 Zang, Ph.D. ${ }^{1}$, Jiangning Chen, Ph.D. ${ }^{1}$, Lei Dong, Ph.D. ${ }^{1}$, Junfeng Zhang, Ph.D. ${ }^{1}$,* \& Zhi Ding, 5 Ph.D. ${ }^{1,2 *}$

6

$7{ }^{1}$ State Key Laboratory of Pharmaceutical Biotechnology, School of Life Sciences, Nanjing

8 University, Xianlin Avenue 163, Nanjing 210023, Jiangsu, China

$9 \quad{ }^{2}$ Collaborative Innovation Center of Chemistry for Life Sciences, Nanjing University, Hankou

10 Road 22, Nanjing 210093, Jiangsu, China

$11 *$ Correspondence to:

12 Junfeng Zhang: Xianlin Avenue 163, Nanjing 210023, Jiangsu, China; email:

13 jfzhang@nju.edu.cn

14 Zhi Ding: Xianlin Avenue 163, Nanjing 210023, Jiangsu, China; e-mail: dingzhi@nju.edu.cn

15 "Contributed equally to the manuscript 


\section{Abstract:}

17 Objective: The skin is an important immunological barrier of the body as well as an optimal route for vaccine administration. Gua Sha, which involves press-stroke treatment of the skin, is an effective folk therapy, widely accepted in East Asia, for various symptoms; however, the mechanisms underlying its therapeutic effects have not been clarified. We investigated the influence of Gua Sha on the immunological features of the skin.

Methods: Gua Sha was performed on BALB/c mice and the effects were evaluated using anatomical, histological, and cytometric methods as well as cytokine determination locally and systemically. The effect on intradermal vaccination was assessed with antigen-specific subtype antibody responses.

Results: Blood vessel expansion, erythrocyte extravasation, and increased ratios of immune active cells were observed in the skin tissue following the treatment. Pro-inflammatory cytokines were up-regulated, and immunosuppressive cytokines, down-regulated, in the treated and untreated skin and systemic circulation; no obvious variations were detected in case of antiinflammatory cytokines. Interestingly, intradermal delivery of a model vaccine following Gua Sha induced about three-fold higher IgG titers with a more Th1-biased antibody subtype profile. Conclusion: Gua Sha treatment can up-regulate the innate and adaptive immune functions of the skin and boost the response against intradermal antigens. Thus, Gua Sha may serve as a safe, inexpensive, and independent physical adjuvant for intradermal vaccination. 


\section{Introduction:}

36

37

People scratch their skin unconsciously in response to an itching sensation despite the fact that it may open and damage wounds at the site and result in infection and even inflammation. Scratching can accelerate the expansion of erythema and exacerbate symptoms in patients with contact urticaria or atopic dermatitis (Wuthrich 1998). With these side effects as tradeoffs, scratching must have some value that allows its existence over time, besides offering relief from itching and the dissipation of invading insects and parasites. Scratching might be able to extend signals locally to the skin and modulate defensive functions. The Chinese invented therapies based on mechanical manipulation of the skin, e.g., Gua Sha and Baguan (Cupping), around 2000 years ago, which are empirically effective towards multiple conditions such as chronic pain, common cold, heatstroke, and respiratory problems. The functions of scratching and the mechanisms underlying the therapeutic effects of Gua Sha encouraged us to undertake this study. Literally, Gua refers to the scratching of the skin, while Sha refers to the petechiae and texture appearing after scratching (Odhav et al. 2013). Gua Sha is defined as repeated, unidirectional, press-stroke of the lubricated skin area with a smooth-edged instrument (Fig 1a \& 1b) until Sha, i.e., blemishes, appear due to blood congestion (Fig 1c). The blemishes fade and completely resolve within 2-5 days in humans with the symptoms getting alleviated immediately or few hours later (Braun et al. 2011). The skin is the largest organ of the body, the interface most exposed to the external environment, and the first-line defense against a broad range of microorganisms. At present, it is clear that the skin serves as a highly sophisticated, potent immune surveillance system related to both innate and adaptive immunity (Weniger \& Glenn 2013). Its immunological functions primarily rely on the Langerhans' cells (LCs) in the 
57 epidermis and the dermal dendritic cells (DCs) in the dermis (Merad et al. 2008), which are

58 involved in antigen capturing, processing, and presentation, during the skin barrier disruption,

59 pathogen invasion, or vaccination; and can cause inflammation, immune activation, or tolerance,

60 depending on various conditions. (Matzinger 2002) (Ding et al. 2010; Engelke et al. 2015).

61 Therefore, the therapeutic mechanism of Gua Sha is believed to be highly relevant for the

62 immunological functions of the skin.

63

[Insert Fig 1 here]

65

To the best of our knowledge, the effects of Gua Sha treatment on the immunological

67 features of the skin have not been clarified. In the current study, it is hypothesized that Gua Shainduced extravasation of blood and controllable skin tissue damage leads to the wound-healing 69 process, including the increase in the level of pro-inflammatory cytokines, and decrease in the 70 level of immunosuppressive cytokines. This results in sensitized innate and adaptive immunity,

71 both locally and systemically. Our studies helped to establish a connection between Gua Sha and 72 the immunological features of the skin. The effect of this treatment on the surface microcirculation in the skin tissue was also confirmed. The skin cytokine levels post-Gua Sha as

74 well as the antibody titers after vaccine administration at the treatment site were determined in 75 preclinical trials. Thus, the effects of Gua Sha on the skin immune system as well as the 76 intradermal vaccination are being studied. 


\section{Materials and Methods:}

78

79

80

81

82

83

84

Materials Aldrich (Shanghai, China). Pentobarbital sodium was obtained from Merck, and Tween 20 from Sangon Biotech Co., Ltd (Shanghai, China). Horseradish peroxidase (HRP)-conjugated goat antimouse IgG ( $\gamma$-chain specific), IgG1 ( $\gamma 1$-chain specific), and IgG2a ( $\gamma 2$ a-chain specific) were purchased from Southern Biotech (Birmingham, USA). A chromogen, 3,3',5,5'tetramethylbenzidine (TMB), and the substrate buffer were purchased from Beyotime B.V. (Shanghai, China). Skim milk powder (Yili) was obtained from the local supermarket. All other chemicals were of analytical grade, and all solutions were prepared with Milli-Q water.

\section{Animals}

Female BALB/c mice $\left(\mathrm{H}-2^{\mathrm{d}}\right), 6-8$ weeks old at the start of the experiments, were purchased from the Experimental Animal Center of Nanjing Medical School (Nanjing, China) and maintained under standardized pathogen-free conditions in the animal facility of the State Key Laboratory of Pharmaceutical Biotechnology, Nanjing University. All mice were housed in standard cages, with free access to food and water. The animals were maintained at a constant temperature $\left(20-21{ }^{\circ} \mathrm{C}\right)$ and humidity $(55 \% \pm 5 \%)$, with a 12 -h light/dark cycle. All animals were handled in accordance with the Declaration of Helsinki, 1975 (as revised in 2008) concerning animal rights, and the protocols were approved by the Institutional Animal Care and Use Committee of Model Animal Research Center of Nanjing University (Ref No. 20130015). Efforts were made to minimize the amount of animals used and ameliorate animal suffering. 
98

99

100

101

102

103

104

105

106

107

108

109

110 111 skin remained intact.

112

113

114

115

116

117 Japan) (Goldner 1938).

\section{Gua Sha treatment on experimental mice}

Mice were anesthetized by intraperitoneal injection of $100 \mathrm{mg} / \mathrm{kg}$ pentobarbital. Gua Sha was performed on the side of the mouse's back by using a smooth-edged instrument made of bull-horn (Fig 1a \& 1d), after the hair was shaved with a clipper a day prior to the experiment. This material was chosen instead of plastic to avoid accumulation of static electricity during operation. The shaved skin area was wiped with $70 \%$ ethanol and left to dry. Then, press-stroke treatment (called "scrape" henceforth) was applied 20 or 40 times in a unidirectional manner, with an angle of $\sim 90^{\circ}$ between the instrument and the mouse's back. The force of scrape was optimized and standardized at $\sim 3 \mathrm{~N}$, so that blemishes would appear between 20 and 40 scrapes, while the inner organs would not be damaged. Twenty scrapes took $\sim 30$ s, and a skin area of $\sim 3$ $\mathrm{cm}^{2}$ was treated. Lubricant oil was not included in the experiments, as the friction was not harmful and the effect of lubricant oil on the skin tissue and vaccine delivery would need to be excluded. No abrasion-induced open wound was visible during and after the treatment, and the

\section{Histological imaging of the treated skin}

Mice were euthanized by cervical dislocation at the indicated time points after Gua Sha treatment. For histopathological examination, samples of treated/untreated skin tissue were obtained, fixed in Bouin's buffer, embedded in paraffin, and sectioned. The sections stained with Masson's trichrome method were analyzed using optical microscopy (Nikon TE2000-U, Tokyo, 
At indicated time points after Gua Sha treatment, the mice were euthanized by cervical dislocation. Approximately $3-\mathrm{cm}^{2}$ skin tissue from the treatment site was excised, chopped to small pieces, and digested in $4 \mathrm{mg} / \mathrm{ml}$ type I collagenase in Hank's balanced salt solution (HBSS,

122 Sigma-Aldrich) at $37^{\circ} \mathrm{C}$ for $2 \mathrm{~h}$. Single cell suspensions were prepared using a 70 - $\mu \mathrm{m}$ cell 123 strainer (BD Bioscience, Shanghai) at the concentration of $1 \times 10^{7} / \mathrm{ml}$ in phosphate-buffered 124 saline (PBS) containing 1\% BSA. Cells were stained using APC-conjugated hamster anti-mouse 125 CD11c, PE-conjugated rat anti-mouse F4/80 (BioLegend), or their corresponding isotype controls. After incubation on ice for $30 \mathrm{~min}$, the cells were washed twice with PBS containing 1\% BSA and re-suspended for flow cytometry analysis using BD FACS Calibur. Data were analyzed using FlowJo software (Tree Star).

Mice were euthanized by cervical dislocation at indicated time points after Gua Sha treatment. Approximately $100 \mathrm{mg}$ of skin tissue from the treatment site was excised and rinsed in cold PBS (at $1 \mathrm{mg} / \mu \mathrm{l}$ ), and then homogenized on ice using Lysing Matrix D tubes and Fast Prepas follows: 1) untreated skin from naïve mice; 2) treated skin samples taken $1 \mathrm{~h}$ after 40 scrapes; 3) untreated skin samples from treated mice $1 \mathrm{~h}$ after 40 scrapes; 4) treated skin samples taken 2 h after 40 scrapes; 5) untreated skin samples from treated mice $2 \mathrm{~h}$ after 40 scrapes. Supernatants were collected after centrifugation at $14,000 \mathrm{rpm}$ for $20 \mathrm{~min}$ at $4{ }^{\circ} \mathrm{C}$. Cytokine levels, including tumor necrosis factor (TNF)- $\alpha$, interleukin (IL)-6, IL-4, IL-5, IL-6, IL-10, IL-12p70, IL-13, and IL-23 of the lysates were quantified using enzyme-linked immunosorbent assay (ELISA) kits, 
140 following the manufacturer's instructions (4A Biotech Co. Ltd. Beijing, China). Blood samples,

141 4-5 drops each time per mouse, were collected from the retro-orbital venous sinus of the mice

1420.5 and $1 \mathrm{~h}$ after 40 scrapes. Blood samples of the untreated mice were collected with the same

143 procedure. Cell-free serum was obtained in collecting tubes with coagulation agent and

144 separation gel (Gongdong, Taizhou, China) by centrifugation after clot formation. TNF- $\alpha$, IL-1 $\beta$,

145 and IL-6 levels in the sera were also determined and compared to those of the untreated mice.

146 Serum nitric oxide level was determined using an assay kit (Jiancheng Bioengineering Institute,

147 Nanjing, China) based on the Griess reaction method, following the manufacturer's instructions

148 (Guevara et al. 1998).

149

150

151

152

153

154

155

156

157

158

159

160

161

162

\section{Immunization and serum antibody assays}

Mice were intradermally (i.d.) immunized with $5 \mu \mathrm{g}$ of OVA under anesthesia three times on day 1, 21, and 42 at a site between the back and thigh 10 min after 20 or 40 scrapes, and euthanized on day 56. Groups of OVA alone and OVA with FIA delivered i.d. to naïve mice were included as the negative and positive controls, respectively. Blood samples were drawn from the tail vein one day before each immunization or from the retro-orbital venous sinus during euthanasia under systemic anesthesia. Cell-free sera were obtained as mentioned above and stored at $-80^{\circ} \mathrm{C}$.

OVA-specific serum IgG, IgG1, and IgG2a titers were determined by ELISA as described previously (Ding et al. 2009). Briefly, ELISA plates (Costar 9018, Shanghai) were coated with OVA at $4^{\circ} \mathrm{C}$ overnight. Two-fold serial dilutions of the serum samples were applied, and the antibodies were detected by HRP-conjugated goat anti-mouse IgG, IgG1, or IgG2a by using TMB as the substrate. Antibody titers are expressed as the calculated sample dilution corresponding to the half of the maximum absorbance at $450 \mathrm{~nm}$ of a complete sigmoid 
163 absorbance-log dilution curve. If the samples were not diluted in the optimal range, additional 164 measurements with more diluted or concentrated samples were performed to complete the $S$ 165 shaped curve. Mice whose serum samples did not reach the half-saturated absorbance value at 166 the lowest (ten-fold) dilution were considered non-responders at that time point and the titers 167 were arbitrarily considered 10.

168 Statistical analysis

169 Antibody titers were logarithmically transformed for better normality. Analysis was 170 performed as indicated. Statistical analysis was carried out using Graphpad (Prism, CA) and a $p$ 171 value of $<0.05$ was considered significant.

172

173 Results:

174 Skin scrapes lead to blood congestion, blood vessel expansion, and infiltration of immune active 175 cells locally

176 Treated skin samples were observed with the naked eye as well as with Masson's staining

177 in order to study the effect of scrapes on the skin. The skin of the naïve mouse after hair removal 178 looked white with a pinkish background. From the dermal side, it was milky white with hardly 179 any capillaries visible (Fig $1 \mathrm{~d} \& 1 \mathrm{~g}$ ). Scrapes were applied 20 or 40 times in a unidirectional 180 manner on the mouse's back. When observed 30 min after treatment, the skin became darker 181 from the stratum corneum side, with a few blood vessels distinguishable from the dermal side 182 after 20 scrapes (Fig 1e \& 1h). After 40 scrapes, petechiae appeared on the stratum corneum side, 183 and subcutaneous microvascular blood extravasation and bruises could be observed from the 
184 dermal side (Fig 1f \& 1i). The vessels in the subcutaneous tissue expanded considerably, with 185 some of them being located closer to the stratum corneum, as shown in the images of Masson's 186 staining of the Gua Sha-treated skin sections (Fig 2). The increased diameter of the vessels 187 indicated an enhanced blood and lymphatic flow, allowing more rapid substance exchange with 188 the interstitial fluid. Red blood cells, probably together with other cell types and contents, 189 dispersed through the ruptured peripheral blood vessels into the dermis and subcutaneous fat 190 tissues, followed by accumulation for hours.

191

192

193

195

[Insert Fig 2 here]

In the Gua Sha-treated skin tissue, the ratios of CD11 ${ }^{+}$cells, including DCs and activated T lymphocytes, and F4/80 macrophages were found to be increased, as observed by flow cytometry analysis (Fig 3). In the untreated mouse skin, CD11 $\mathrm{c}^{+}$cells accounted for $\sim 4 \%$ of the total cell population. This proportion increased to $\sim 9 \%, 12 \%$, and $14 \%$ at 15,30 , and $60 \mathrm{~min}$ after treatment, respectively (Fig 3a \& 3b). A similar trend was observed in case of macrophages present in the treated skin tissue; the proportion of macrophages increased with time after Gua Sha treatment, from $16.5 \%$ to $\sim 20 \%$ (Fig 3c \& 3d), probably because of the infiltration from the expanded or ruptured blood vessels.

\section{Skin scrapes cause variations in the local and systemic levels of cytokines and nitric oxide}

As Gua Sha treatment enhances microcirculation, more rapid substance exchange occurs among the blood, lymphatic fluid, interstitial fluid, and the infiltrated immune active cells. This 
207 changes the microenvironment of the treated skin tissue. The levels of the representative

208 cytokine groups present locally in the treated skin tissue and systemically in the untreated skin

209 area were determined and compared with those of naïve mice (Fig 4). Local concentrations of

210 most pro-inflammatory cytokines examined, including TNF- $\alpha$, IL-6, IL-12p70, and IL-23,

211 increased moderately, but a significant increase was observed after the treatment, except IL-1 $\beta$.

212 Among them, TNF- $\alpha$ level increased in both treated and untreated skin tissues, while IL-6, IL-

213 12p70, and IL-23 levels in the untreated skin area of the treated mice remained constant. The

214 immunosuppressive cytokine IL-10 was present at lower levels in the treated skin tissue 1 and 2

$215 \mathrm{~h}$ after treatment, and in the untreated skin area $2 \mathrm{~h}$ after treatment, compared to that in the

216 untreated mice, indicating an overall up-regulation of immune reactivity (Fig 4d). The levels of

217 anti-inflammatory cytokines IL-4, IL-5, and IL-13 in the skin tissues of treated and untreated

218 mice were also examined, but no remarkable differences were detected (Fig 1s). In the serum

219 samples of the treated mice, the levels of TNF- $\alpha$, IL-1 $\beta$, and IL-6 increased significantly,

220 compared to those from untreated mice (Fig 5a, 5b, \& 5c). Consistent with the anatomical and

221 histological observations, nitric oxide content, which leads to vasodilation and increased blood

222 flow, was significantly up-regulated (Fig 5d). In addition to increasing the infiltration of immune

223 active cells such as neutrophils, monocytes, and macrophages from the blood, nitric oxide

224 enhances immune defense by acting as a free radical with oxidative pressure and is toxic to

225 bacteria and intracellular parasites.

226

227

[Insert Fig 4 here]

228

[Insert Fig 5 here] 
232 hypothesized that the Gua Sha treatment would enhance adaptive immunity against intradermal 233 pathogens. An in vivo vaccination study was performed to examine its effect on the immune defense of the skin. Intradermal injection, instead of dermal application, was chosen for vaccine administration to ensure exact vaccine dosage. The OVA-specific isotype antibody titers of the serum samples from three different time points were determined (Fig 6). The OVA-specific IgG titers of the Gua Sha-treated groups did not differ significantly from those of the untreated group after prime (Fig 6a). Interestingly while as expected, the systemic humoral response to OVA was augmented, as evidenced by the IgG titers after the first and second booster doses (Fig 6b \& 6c) and IgG1 titer after second booster dose (Fig 6d). 20 and 40 scrapes of the skin induced about two and three fold higher IgG titers, respectively after the second booster dose. In the untreated group, $\operatorname{IgG} 2 \mathrm{a}$ induction was not very pronounced because half of the animals had titers below the detection limit. However remarkably, mice who received Gua Sha treatment before vaccination had elevated IgG2a titers, without any non-responders (Fig 6e). The IgG1/IgG2a ratios of the treated groups after the second booster dose were lower than those of the untreated group (Fig 6f), indicating that Gua Sha treatment of the skin prior to intradermal vaccination may lead to a more Th1-biased immune response. 


\section{Discussion:}

252 Gua Sha is one of the many old empirical practices, which are widely accepted as

253 effective therapies, but the scientific understanding of its mechanisms is lacking. In traditional

254 Chinese medical theory, it is briefly described as a therapy to help expel the toxicant, i.e., Sha,

255 which cannot be cleared and excreted by physiological processes. However, Sha does not

256 directly correspond to known biological substances. Articles about Gua Sha treatment published

257 in the Western medical literature are mainly case reports describing its therapeutic impacts as

258 well as complications, while only a handful of studies discuss its physiological impacts and

259 underlying mechanisms (Kwong et al. 2009; Lauche et al. 2012; Nielsen 2009). This effort is

260 continued in the current study, and it is, to the best of our knowledge, for the first time, that Gua

261 Sha is shown to be able to enhance the humoral immune response, generating higher antibody

262 titers against vaccines applied i.d. on treated skin. It provides direct evidence that scratching on

263 the skin as well as Gua Sha treatment can serve as a mechanical signal to enhance the immune

264 surveillance function of the skin.

265

Although Gua Sha treatment is relatively simple, it generates multiple stimuli to the skin.

266 The effect of Gua Sha on immunity may be a synergistic result attributed to the following factors.

267 First, the mechanical press-stroke movement enhances surface microcirculation in the skin tissue

268 and induces the expansion of capillaries, including the blood vessels and lymphatic vasculature

269 (Nielsen et al. 2007). The increased blood and lymphatic flow leads to more rapid substance and

270 cell exchange among blood, interstitial fluid, and lymphatic fluid. As the afferent lymphatic

271 vessels are the route through which DCs migrate to the lymph nodes and lymphoid organs after

272 antigen uptake, this, in turn, promotes the infiltration and migration of DCs and other immune 
273 active cells and accelerates the initialization of humoral immune response against the incoming

274 antigens (Wang \& Oliver 2010). Second, the extravasation of blood and blood components, i.e.,

275 red blood cells and platelets, into the subcutis can induce multiple effects on immunity and

276 inflammation (McFadyen \& Kaplan 2015), leading to a fluctuation in cytokine levels and

277 initialization of the wound-healing process. Given the varied levels of cytokines at the local site

278 after Gua Sha treatment, e.g. higher levels of Th1-biased immunopotentiators, namely, TNF- $\alpha$,

279 IL-6, and IL-12 (Afonso et al. 1994; Singh et al. 2011; Tovey \& Lallemand 2010) and lower

280 levels of the immunosuppressive IL-10, the antigen-presenting cells would be more rapidly

281 activated and could lead to a stronger and more Th1-biased humoral response, as confirmed in

282 the present study. In addition, Gua Sha generates temporary warming effects on the skin tissue,

283 like short-term and local fever. We suggest that this thermal element of Gua Sha might also

284 contribute in stimulating the innate and adaptive immune responses (Evans et al. 2015).

285 Gua Sha treatment creates controllable tissue damage and low-scale inflammation in the

286 skin tissue, with a remarkable profile of changes in the cytokine levels. It prepares the

287 surrounding skin tissues for possible pathogenic challenges and induces an immune defense

288 exercise. Similar processes, which cause moderate tissue damage, such as the use of a bifurcated

289 needle for smallpox vaccination (Rubin 1980), microneedle arrays (Fernando et al. 2010), tape-

290 stripping (Inoue \& Aramaki 2007), non-ablative fractional laser (Wang et al. 2015), low-

291 frequency ultrasound (Tezel et al. 2005), and thermal ablation (Garg et al. 2007), have been

292 reported to improve (trans)dermal vaccination to various extends. These are caused not only by

293 tissue damage, but also skin barrier disruption, hence, increased antigen delivery. In contrast, in

294 our study, i.d. vaccination of the same antigen dose clearly showed that tissue damage caused by

295 Gua Sha treatment is the direct cause of the adjuvanticity observed. Although not determined in 
296 this study, Gua Sha treatment may as well compromise the skin barrier function. Standardized

297 Gua Sha treatment with controllable impairment of the skin barrier may facilitate needle-free

298 dermal vaccine delivery and improve its efficacy.

299 Gua Sha has been applied in human for centuries with strong safety records and it 300 involves very simple instruments. However, Gua Sha therapies differing in the application force,

301 directions, patterns, and inclusion of oils have been developed to treat different diseases and 302 symptoms. A more elaborate and robust experimental design involving higher animals or human 303 beings is, therefore, needed to unravel more about the underlying mechanisms. In practice, it 304 could be applied prior to vaccination as a physical adjuvant, especially for those with poorly 305 functioning immune systems. It needs to be pointed out that the ratios of treated/total skin area in 306 the mice in this study are certainly higher than those in humans, and therefore, it may not be as 307 effective as in mice. However, it is a safe, inexpensive, and versatile method, which can be employed separately or in combination with other biochemical adjuvants, without concerns about antigen-adjuvant or adjuvant-adjuvant interactions. As far as the mechanism of adjuvanticity is concerned, Gua Sha treatment may also boost the immune response to the vaccines delivered in the untreated skin area, subcutaneously or at the mucosal surface, and help convert the nonresponders to certain vaccines, a domain that needs to be further studied.

\section{Conclusions:}

We conclude that Gua Sha treatment can improve the immunological functions of the skin and body by acting as a physical adjuvant, as shown by the increased ratio of immune active cells, variation in cytokine levels, and enhanced Th1-biased humoral immunity to intradermal vaccination at the site of treatment. 


\section{References:}

319 Afonso L, Scharton T, Vieira L, Wysocka M, Trinchieri G, and Scott P. 1994. The adjuvant 320 effect of interleukin-12 in a vaccine against Leishmania major. Science 263:235-237. 10.1126/science.7904381

322 Braun M, Schwickert M, Nielsen A, Brunnhuber S, Dobos G, Musial F, Ludtke R, and

Ding Z, Verbaan FJ, Bivas-Benita M, Bungener L, Huckriede A, van den Berg DJ, Kersten G, and Bouwstra JA. 2009. Microneedle arrays for the transcutaneous immunization of diphtheria and influenza in BALB/c mice. J Control Release 136:71-78. S01683659(09)00079-0 [pii]. 10.1016/j.jconrel.2009.01.025

Engelke L, Winter G, Hook S, and Engert J. 2015. Recent insights into cutaneous immunization: How to vaccinate via the skin. Vaccine 33:4663-4674. 10.1016/j.vaccine.2015.05.012

Evans SS, Repasky EA, and Fisher DT. 2015. Fever and the thermal regulation of immunity: the immune system feels the heat. Nat Rev Immunol 15:335-349. 10.1038/nri3843

Fernando GJ, Chen X, Prow TW, Crichton ML, Fairmaid EJ, Roberts MS, Frazer IH, Brown LE, and Kendall MA. 2010. Potent immunity to low doses of influenza vaccine by 
probabilistic guided micro-targeted skin delivery in a mouse model. PLOS ONE 5:e10266.

341

Garg S, Hoelscher M, Belser JA, Wang C, Jayashankar L, Guo Z, Durland RH, Katz JM, and Sambhara S. 2007. Needle-free skin patch delivery of a vaccine for a potentially pandemic influenza virus provides protection against lethal challenge in mice. Clin Vaccine Immunol 14:926-928.

Goldner J. 1938. A modification of the masson trichrome technique for routine laboratory purposes. Am J Pathol 14:237-243.

Guevara I, Iwanejko J, Dembinska-Kiec A, Pankiewicz J, Wanat A, Anna P, Golabek I, Bartus S, Malczewska-Malec M, and Szczudlik A. 1998. Determination of nitrite/nitrate in human biological material by the simple Griess reaction. Clin Chim Acta 274:177-188.

Inoue J, and Aramaki Y. 2007. Toll-like receptor-9 expression induced by tape-stripping triggers on effective immune response with CpG-oligodeoxynucleotides. Vaccine 25:1007-1013.

Kwong KK, Kloetzer L, Wong KK, Ren JQ, Kuo B, Jiang Y, Chen YI, Chan ST, Young GS, and Wong ST. 2009. Bioluminescence imaging of heme oxygenase-1 upregulation in the Gua Sha procedure. J Vis Exp. 10.3791/1385

Lauche R, Wubbeling K, Ludtke R, Cramer H, Choi KE, Rampp T, Michalsen A, Langhorst J, and Dobos GJ. 2012. Randomized controlled pilot study: pain intensity and pressure pain thresholds in patients with neck and low back pain before and after traditional East Asian "gua sha" therapy. Am J Chin Med 40:905-917. 10.1142/S0192415X1250067X

Matzinger P. 2002. The danger model: a renewed sense of self. Science 296:301-305. 10.1126/science.1071059. 296/5566/301 [pii] 
361 McFadyen JD, and Kaplan ZS. 2015. Platelets are not just for clots. Transfus Med Rev 29:110-

$362 \quad 119.10 .1016 /$ j.tmrv.2014.11.006

363 Merad M, Ginhoux F, and Collin M. 2008. Origin, homeostasis and function of Langerhans cells 364 and other langerin-expressing dendritic cells. Nat Rev Immunol 8:935-947. nri2455 [pii].

365 $10.1038 /$ nri2455

366

367

368

369

370

371

372

373

374

375

376

377

378

379

380

381

382

Nielsen A. 2009. Gua sha research and the language of integrative medicine. J Bodyw Mov Ther 13:63-72. 10.1016/j.jbmt.2008.04.045

Nielsen A, Knoblauch NT, Dobos GJ, Michalsen A, and Kaptchuk TJ. 2007. The effect of Gua Sha treatment on the microcirculation of surface tissue: a pilot study in healthy subjects. Explore (NY) 3:456-466. 10.1016/j.explore.2007.06.001

Odhav A, Patel D, Stanford CW, and Hall JC. 2013. Report of a case of Gua Sha and an awareness of folk remedies. Int $J$ Dermatol 52:892-893. 10.1111/j.13654632.2011.05063.x

Rattanapak T, Birchall JC, Young K, Kubo A, Fujimori S, Ishii M, and Hook S. 2014. Dynamic visualization of dendritic cell-antigen interactions in the skin following transcutaneous immunization. PLoS ONE 9:e89503. 10.1371/journal.pone.0089503

Rubin BA. 1980. A note on the development of the bifurcated needle for smallpox vaccination. WHO Chron 34:180-181.

Singh V, Jain S, Gowthaman U, Parihar P, Gupta P, Gupta UD, and Agrewala JN. 2011. Coadministration of IL-1+IL-6+TNF-alpha with Mycobacterium tuberculosis infected macrophages vaccine induces better protective $\mathrm{T}$ cell memory than BCG. PLoS ONE 6:e16097. 10.1371/journal.pone.0016097 
383 Tezel A, Paliwal S, Shen Z, and Mitragotri S. 2005. Low-frequency ultrasound as a 384 transcutaneous immunization adjuvant. Vaccine 23:3800-3807.

385 Tovey M, and Lallemand C. 2010. Adjuvant Activity of Cytokines. In: Davies G, ed. Vaccine 386 Adjuvants: Humana Press, 287-309.

387 Wang J, Li B, and Wu MX. 2015. Effective and lesion-free cutaneous influenza vaccination. $388 \quad$ Proc Natl Acad Sci U S A 112:5005-5010. 10.1073/pnas.1500408112

389 Wang Y, and Oliver G. 2010. Current views on the function of the lymphatic vasculature in 390 health and disease. Genes Dev 24:2115-2126. 10.1101/gad.1955910

391 Weniger BG, and Glenn GM. 2013. Cutaneous vaccination: antigen delivery into or onto the skin. Vaccine 31:3389-3391. 10.1016/j.vaccine.2013.05.048

393 Wuthrich B. 1998. Food-induced cutaneous adverse reactions. Allergy 53:131-135. 


\section{Figure 1}

Introduction of Gua Sha and representative anatomic images of Gua Sha treatment on mouse skin.

Fig 1. Introduction of Gua Sha and representative anatomic images of Gua Sha treatment on mouse skin. (a) The smooth-edged instrument made of bull-horn for Gua Sha treatment, with the size and shape similar as a credit card. (b) Gua Sha treatment on a person's back. (c) The pattern of blemishes resulted from Gua Sha treatment in human. Mouse skin prior to Gua Sha treatment observed from the stratum corneum side (d), (blue arrow indicates the direction of Gua Sha operation) and the dermal side (g); mouse skin after 20 or 40 scrapes observed from the stratum corneum side (e \& f) and the dermal side ( $\mathrm{h} \& \mathrm{i})$. Photos were taken 30 minutes after Gua Sha treatment from the stratum corneum side, then the mice were euthanized for observation from the dermal side. Images are representative ones from three mice per group. 
a.

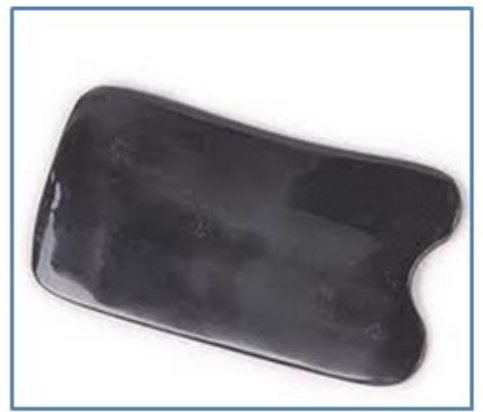

d.

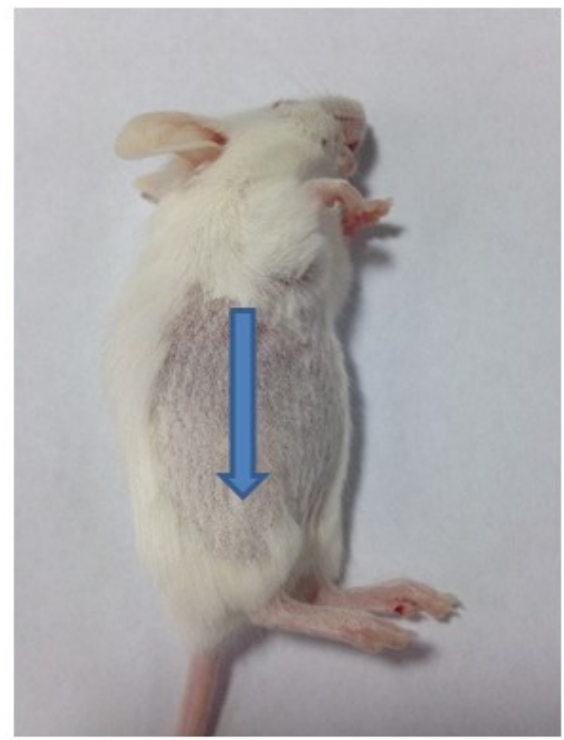

g.

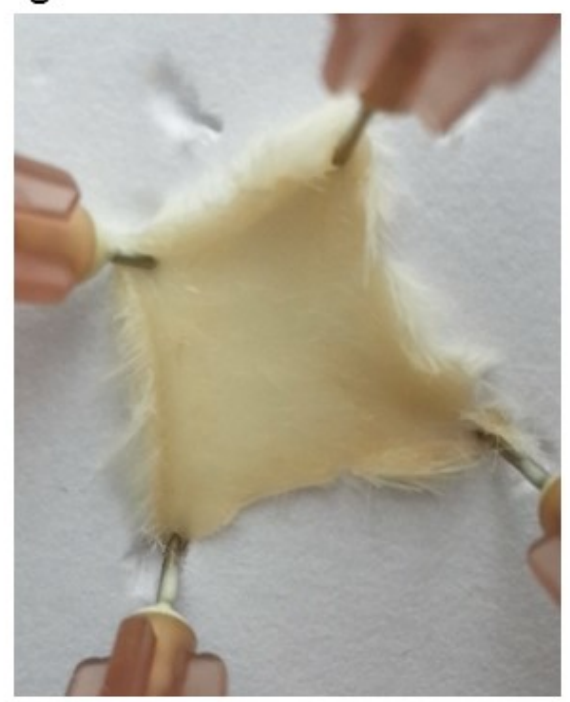

b.

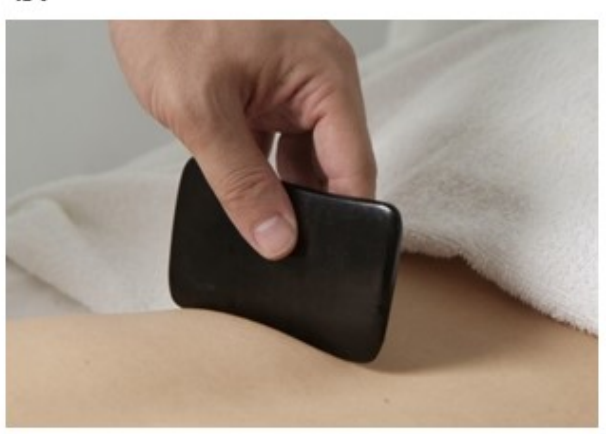

e.

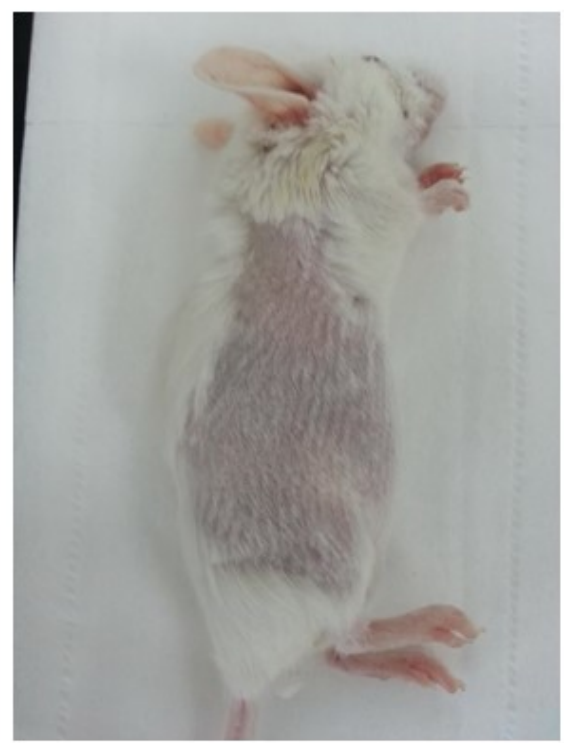

h.

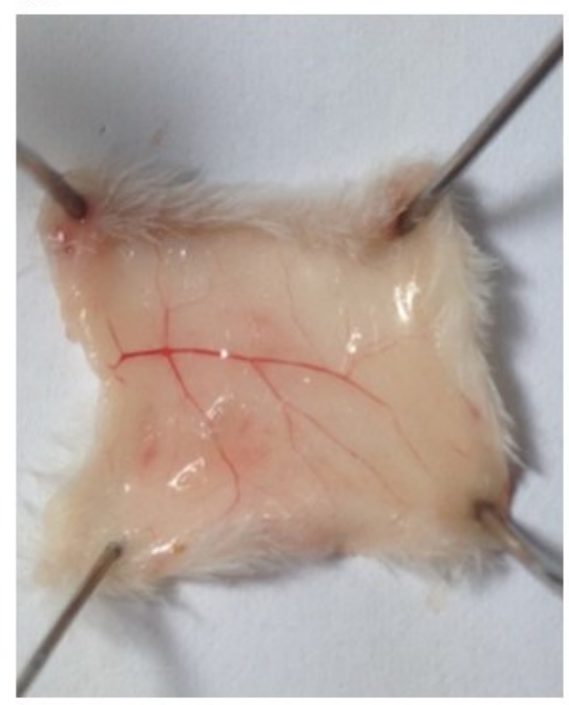

c.

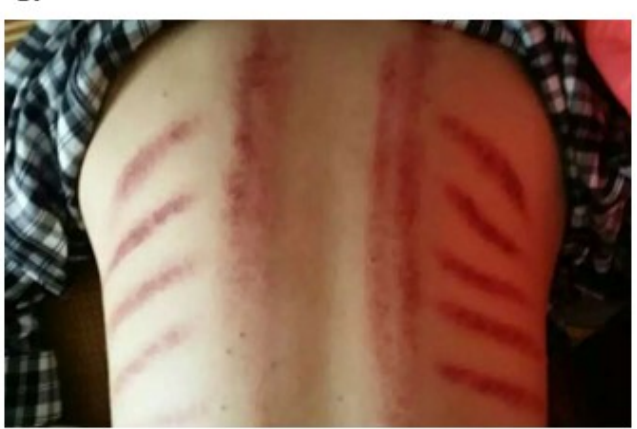

f.

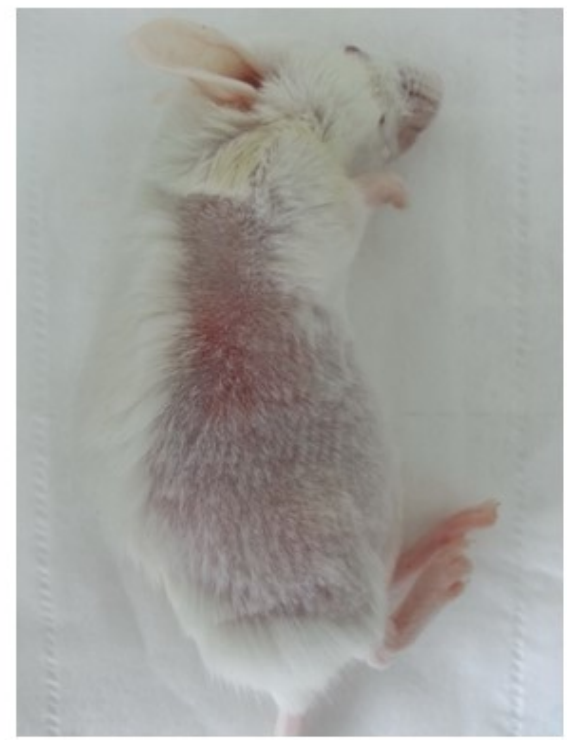

i.

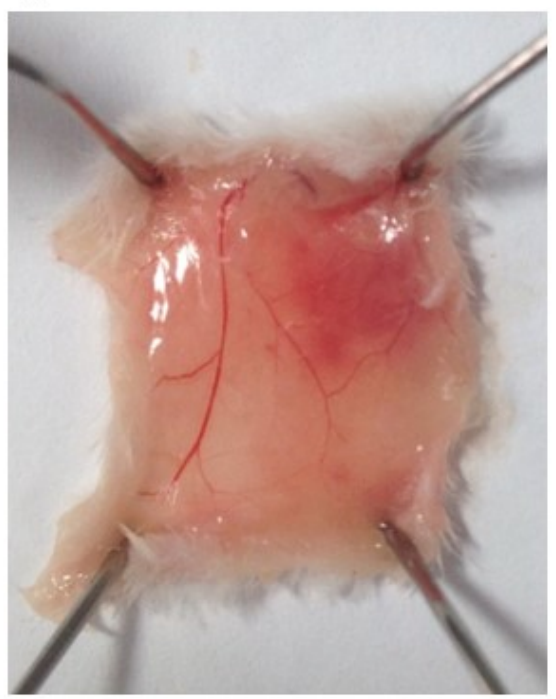




\section{Figure 2}

Histological images of mouse skin sections prior to and after Gua Sha treatment.

Fig 2. Histological images of mouse skin sections prior to and after Gua Sha treatment.

Representative images of Masson's trichrome staining of mouse skin sections prior to and after 40 scrapes at different time points and magnifications were shown from three mice per group, scale bar $=100 \mu \mathrm{m}$. As an example, in the image (100x) taken $15 \mathrm{~min}$ after Gua Sha treatment, the boundaries between epidermis, dermis and subcutaneous tissue were depicted with white dots, and two groups of extravasated red blood cells were pointed out with white circles and arrowheads in dermis and subcutaneous tissue.

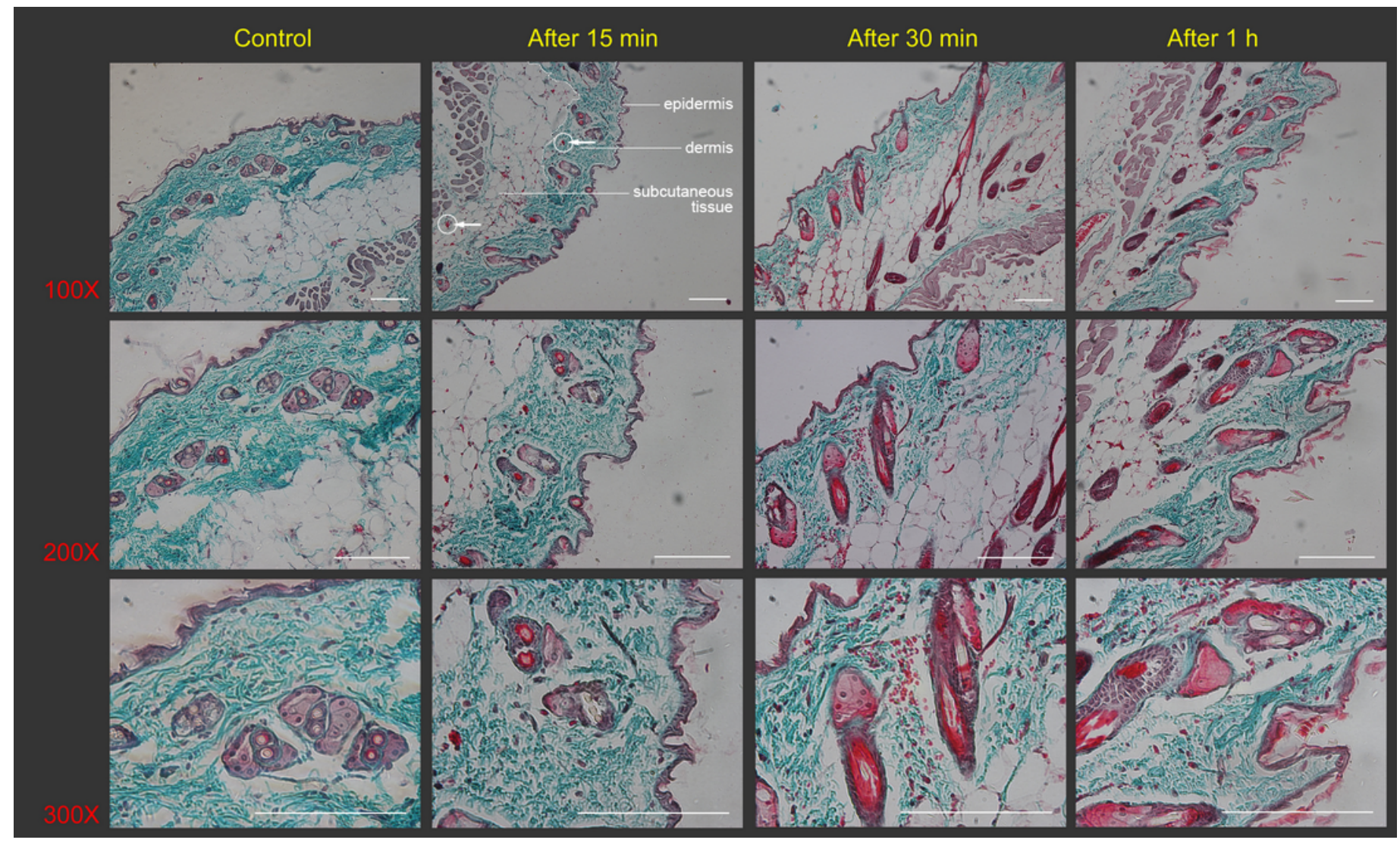




\section{Figure 3 (on next page)}

Flow cytometry analysis of Gua Sha-treated skin tissue.

Fig 3. Flow cytometry analysis of Gua Sha-treated skin tissue. Single cell suspensions from Gua Sha-treated mouse skin or untreated control were prepared and stained with APCconjugated anti-mouse CD11C and PE-conjugated anti-mouse F4/80. Representative

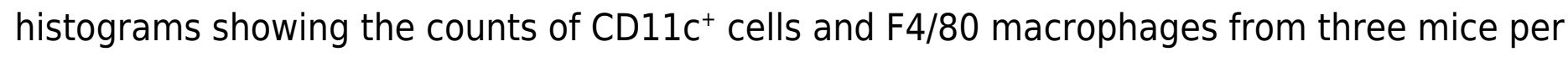
group were shown in (a) \& (c), and the percentages of these cell populations were plotted in (b) $\&(d)$, respectively. Data were shown as Mean + SD. Statistical comparisons were made between each treated group and the untreated group. $(n=3 ; *: p<0.05 ; * *: p<0.01$; ***: $p<0.001$; One-way ANOVA with Dunnett's posttest.) 
a.

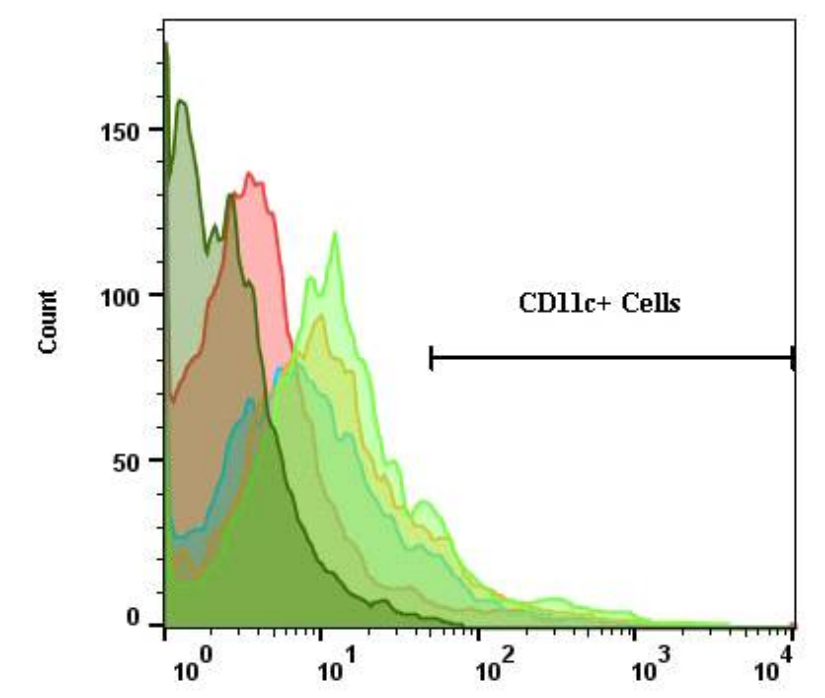

C.

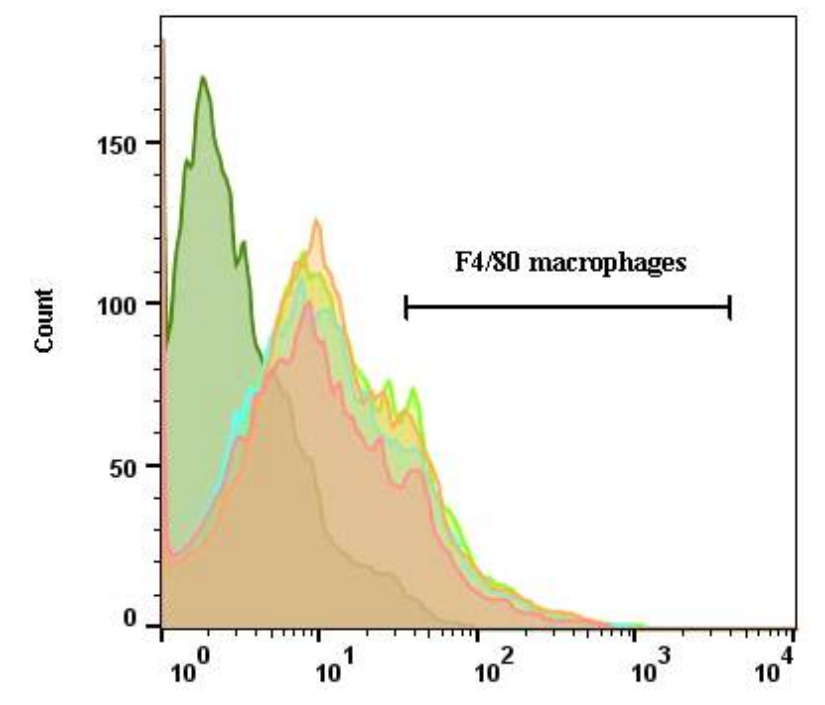

Manuscript to $\mathbf{b}$. reviewed

\begin{tabular}{|c|l|c|}
\hline & Sample & Freq. of CDllc+ Cells (\%) \\
\hline$\square$ & isotype control & 0.43 \\
\hline$\square$ & $60 \mathrm{~min}$ & 14.2 \\
\hline$\square$ & $30 \mathrm{~min}$ & 12.5 \\
\hline$\square$ & $15 \mathrm{~min}$ & 9.32 \\
\hline$\square$ & untreated & 4.18 \\
\hline
\end{tabular}

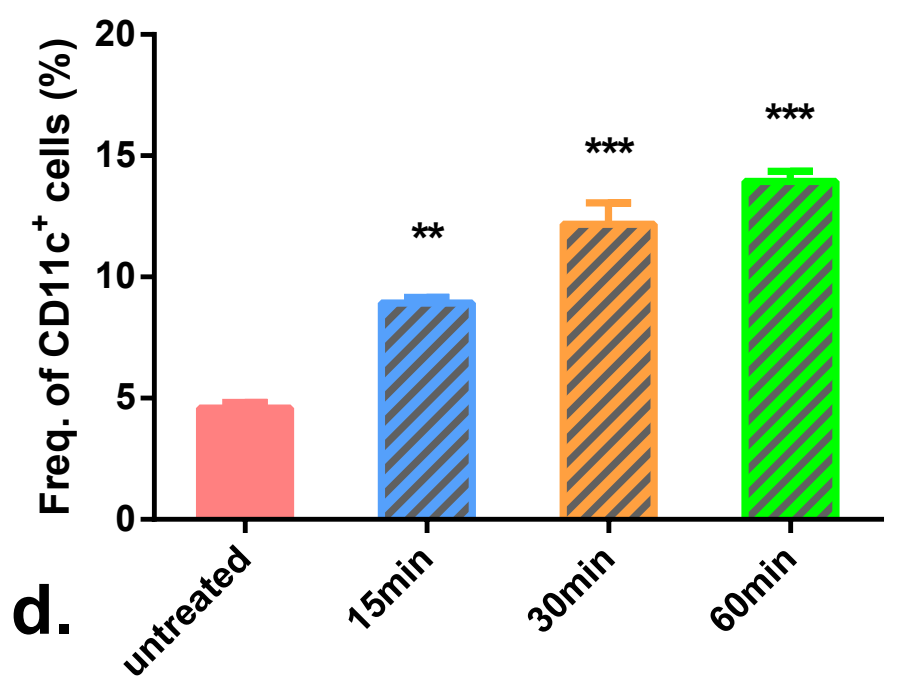

\begin{tabular}{|c|l|c|}
\hline & Sample & Freq. of F4/80 macrophages (\%) \\
\hline$\square$ & isotype control & 1.54 \\
\hline$\square$ & $60 \mathrm{~min}$ & 19.5 \\
\hline$\square$ & $30 \mathrm{~min}$ & 18.4 \\
\hline$\square$ & $15 \mathrm{~min}$ & 17.1 \\
\hline$\square$ & untreated & 15.7 \\
\hline
\end{tabular}

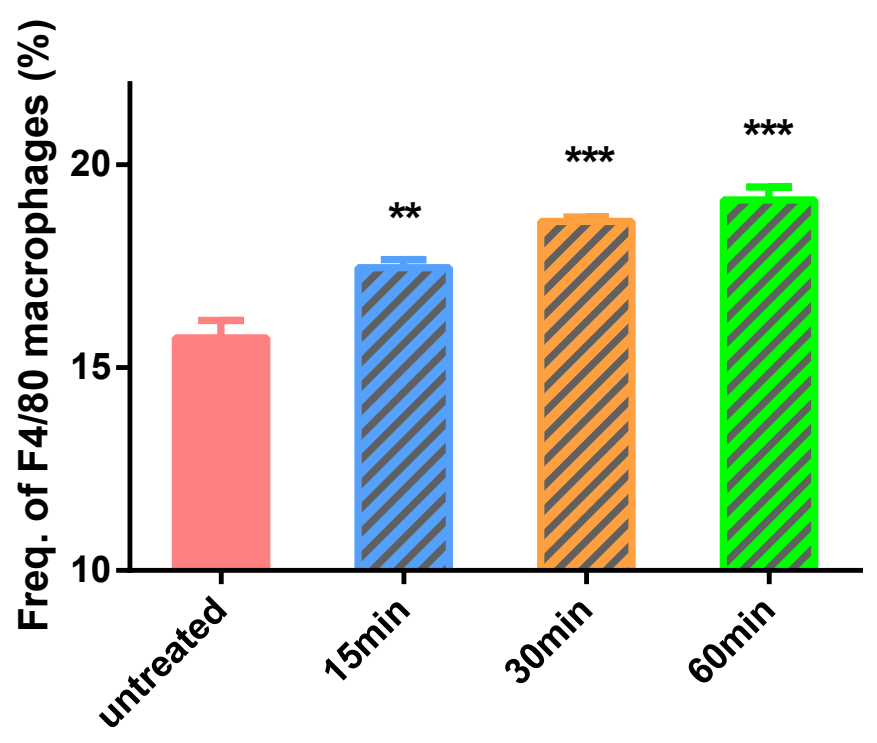




\section{Figure 4 (on next page)}

Levels of cytokines from skin tissues with or without 40 scrapes Gua Sha treatment.

Fig 4. Levels of cytokines from skin tissues with or without 40 scrapes Gua Sha treatment. Groups were set as following: 1) untreated skin from naïve mice; 2 ) treated skin samples taken $1 \mathrm{~h}$ after 40 scrapes; 3) untreated skin samples from treated mice $1 \mathrm{~h}$ after 40 scrapes; 4) treated skin samples taken $2 \mathrm{~h}$ after 40 scrapes; 5) untreated skin samples from treated mice $2 \mathrm{~h}$ after 40 scrapes. $0.1 \mathrm{~g}$ of skin tissue were excised, rinsed in $1 \mathrm{ml} \mathrm{PBS}$ and homogenized on ice. The concentrations of TNF- $\alpha$ (a), IL-1ß (b), IL-6 (c), IL-10 (d), IL-12p70 (e), and IL-23 (f) in the supernatants were measured with ELISA and data were shown as mean + SD . Statistical comparisons were made between each group and the untreated group. ( $\mathrm{n}=6, *: p<0.05 ; * *: p<0.01 ; * * *: p<0.001$; One-way ANOVA with Dunnett's posttest.) 
a.

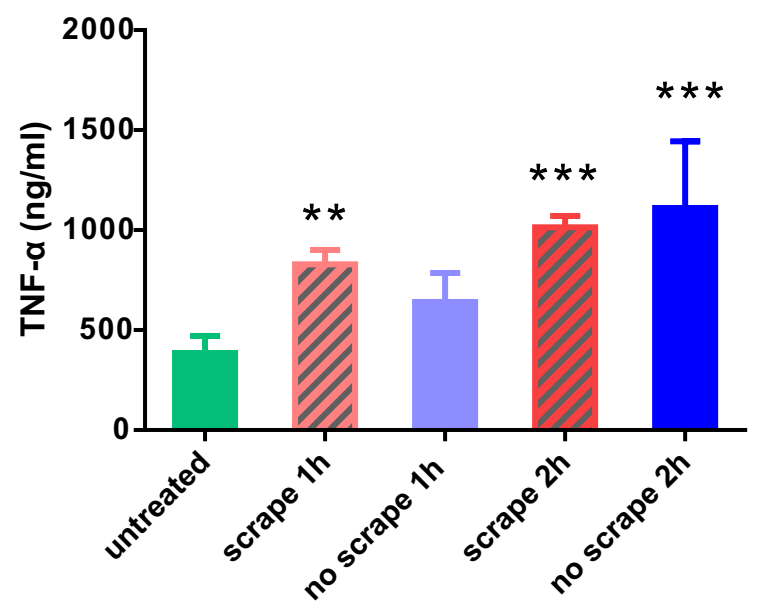

Mouse groups $(n=6)$

C.

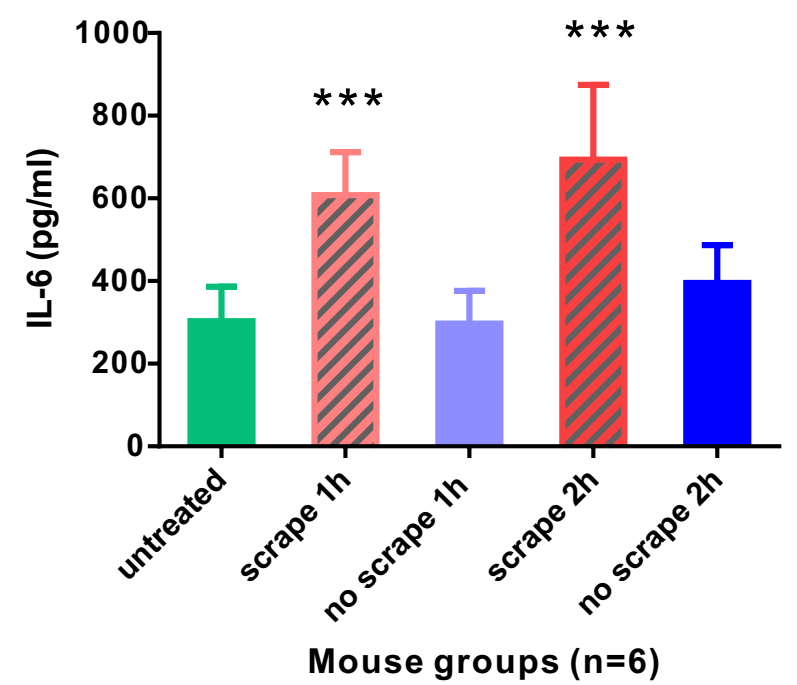

e.

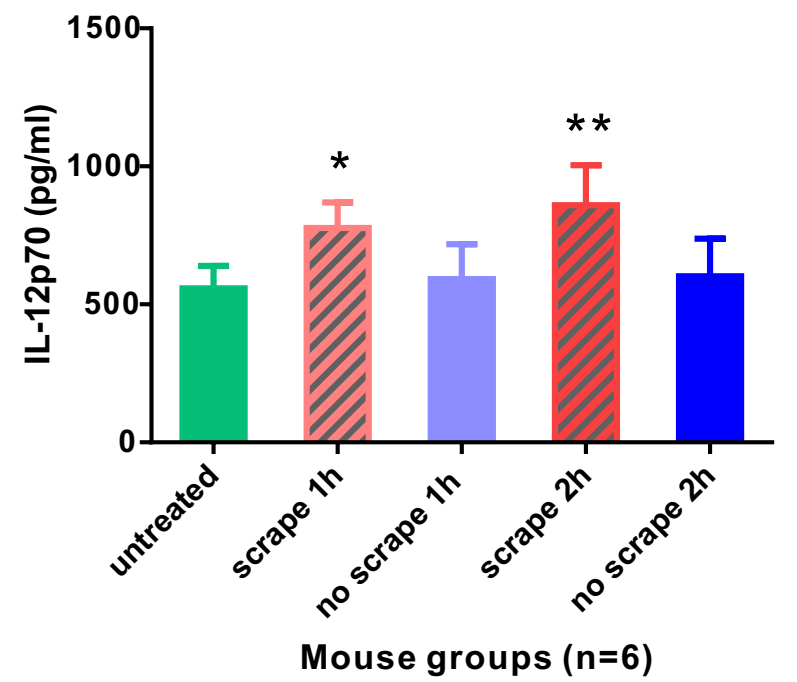

b.

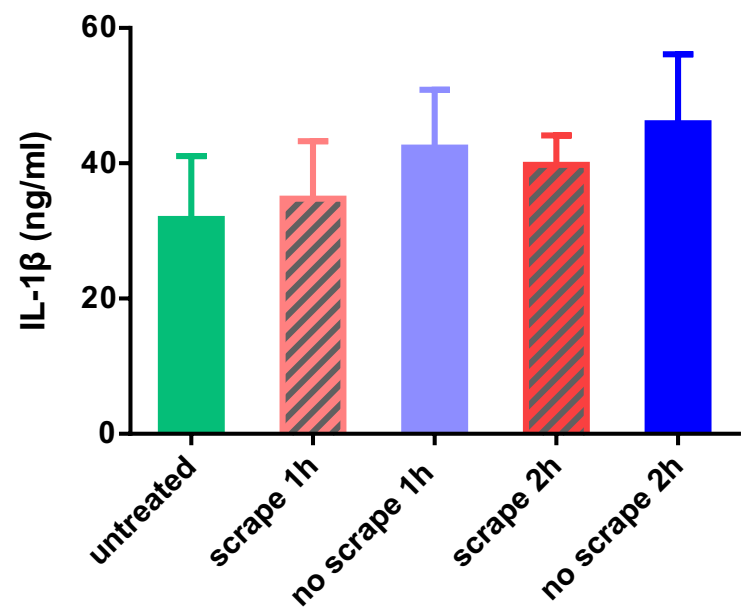

Mouse groups $(n=6)$

d.

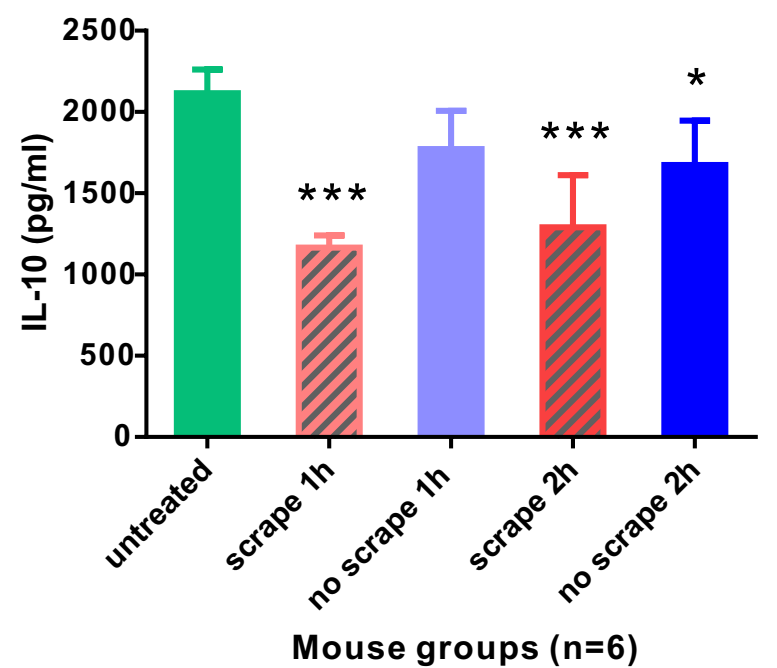

f.

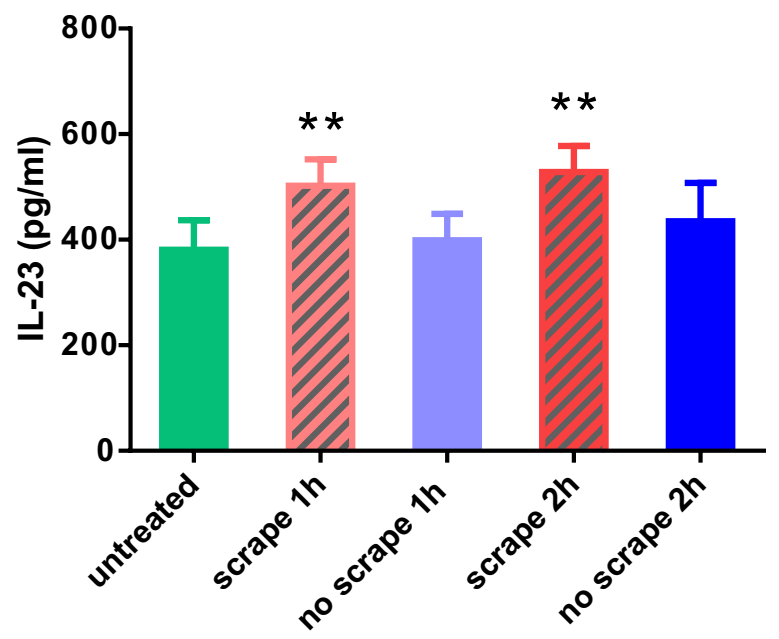

Mouse groups $(n=6)$ 


\section{Figure 5 (on next page)}

Levels of pro-inflammatory cytokines and nitric oxide from serum samples.

Fig 5. Levels of pro-inflammatory cytokines and nitric oxide from serum samples. Blood samples were taken 0.5 and 1 hour after 40 scrapes Gua Sha treatment. Samples taken from untreated mice with identical blood-taking regime were as the control. The concentrations of TNF- $\alpha(a), I L-1 \beta$ (b), and IL-6 (c) were measured with ELISA and the content of nitric oxide was determined using the nitrate reductase method (d). Data were shown as mean + SD. Statistical comparisons were made between the treated and untreated groups. $(n=6, *$ : $p<0.05 ; * * *: p<0.001$; Two-way ANOVA with Bonferroni's posttest.) 
a.

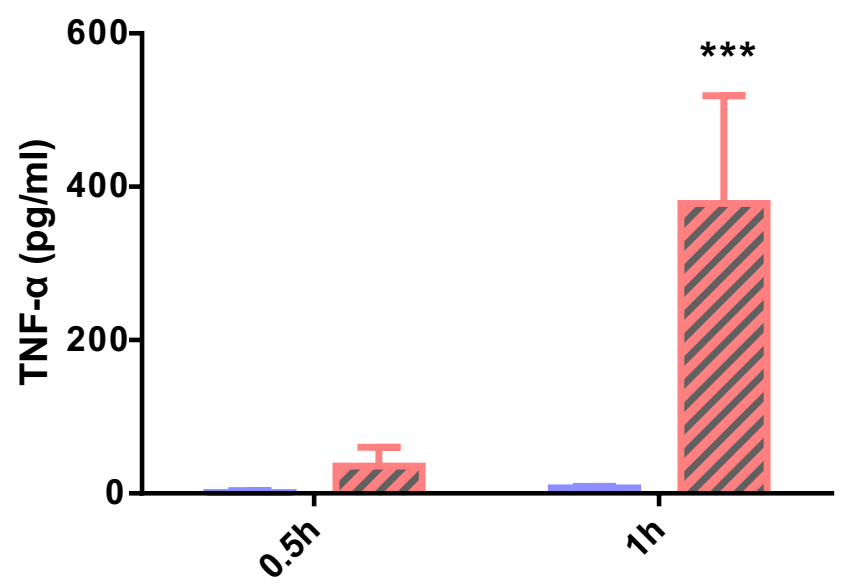

C.

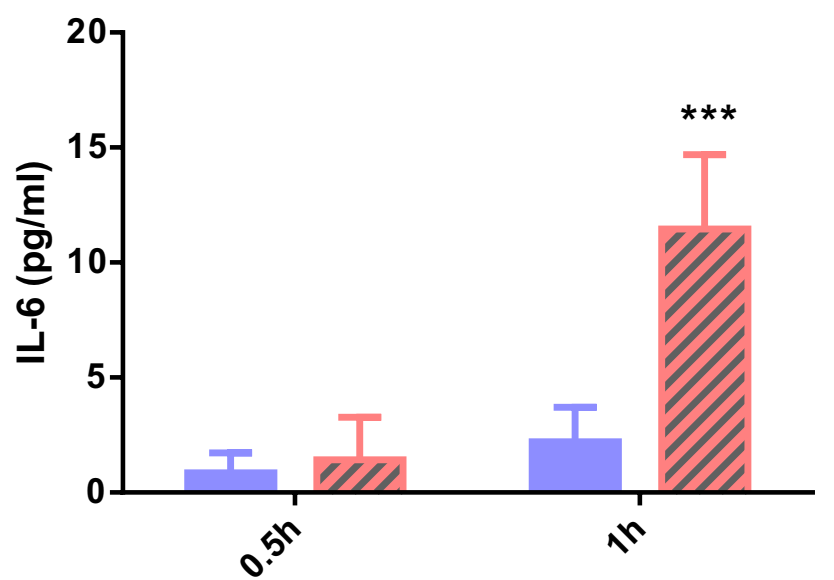

b.

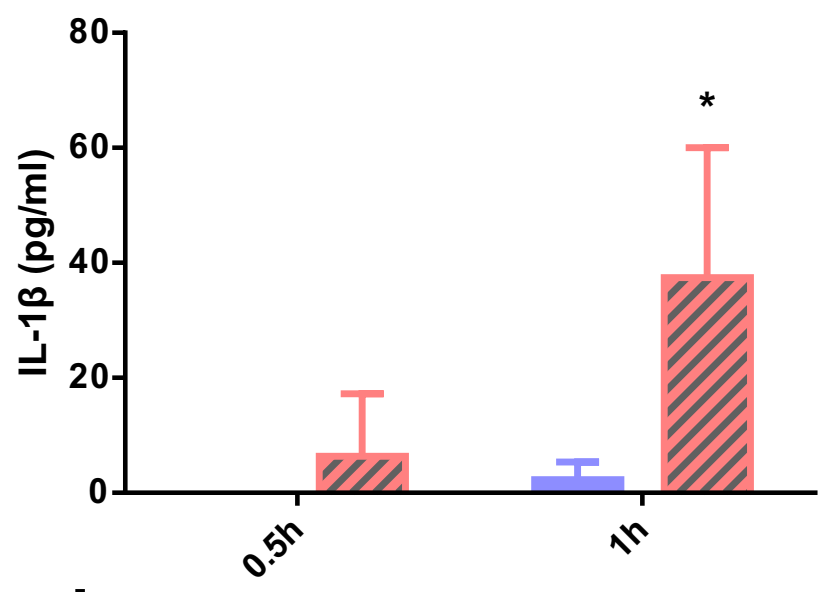

d.

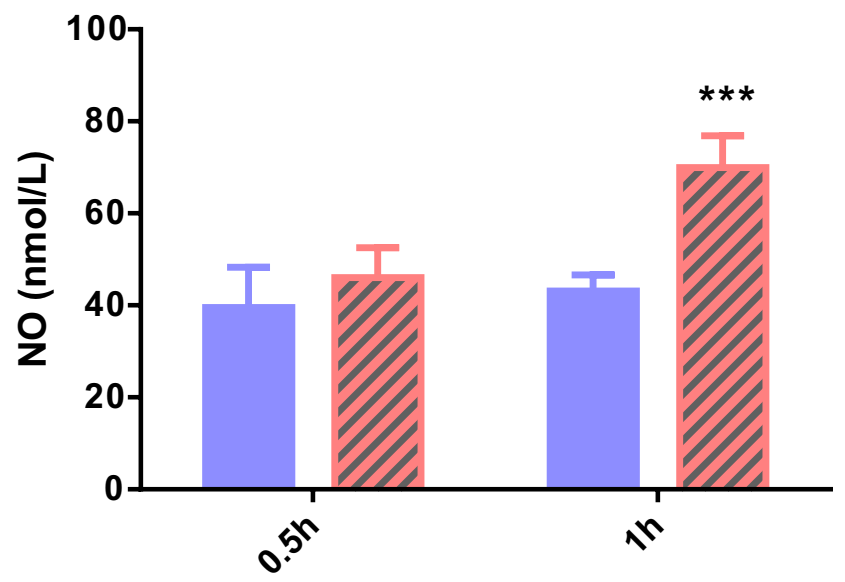




\section{Figure 6 (on next page)}

Serum OVA-specific IgG subtype antibody titers after i.d.[i] immunization.

Fig 6. Serum OVA-specific IgG subtype antibody titers after i.d. immunization. Vaccination was performed $10 \mathrm{~min}$ after 20 or 40 scrapes at day 1, 21 and 42. Groups vaccinated with OVA and OVA+FIA in untreated mice were as the negative and positive controls, respectively. Sera were collected after prime, the first boost and the second boost (day 20, 41 and 55). IgG $(a, b$ and $c), \lg G 1$ (d) and IgG2a (e) were determined with ELISA. Data were shown as mean \pm SD. OVA-specific $\operatorname{lgG1/lgG2a}$ ratios of individual mouse were calculated using IgG2a responders. Data were shown as mean $+\mathrm{SD}$ (f) $(\mathrm{n}=6 ; *: p<0.05 ; * *: p<0.01 ; * * *: p<0.001$; One-way ANOVA with Dunnett's posttest.) 

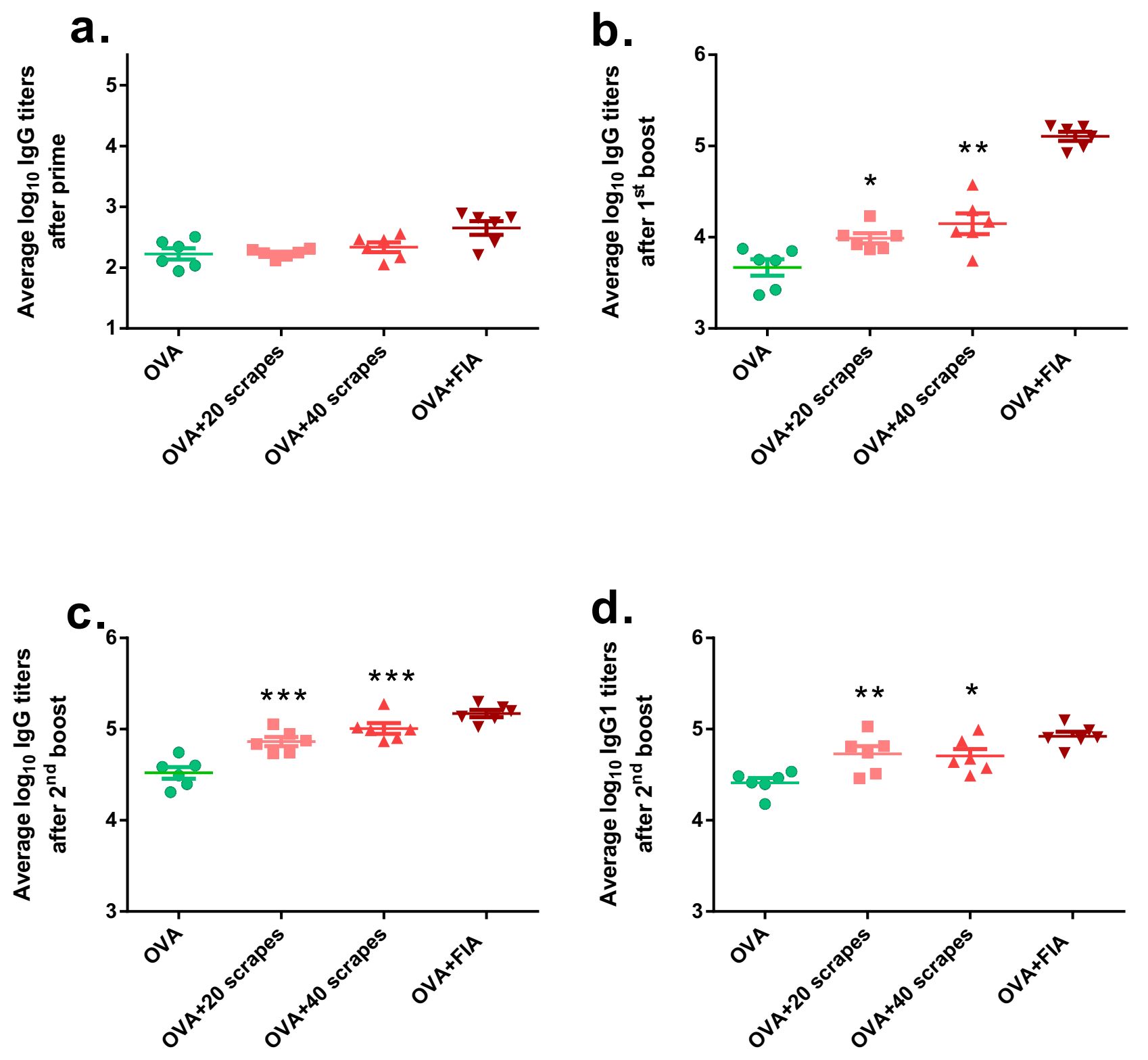

e.

f.
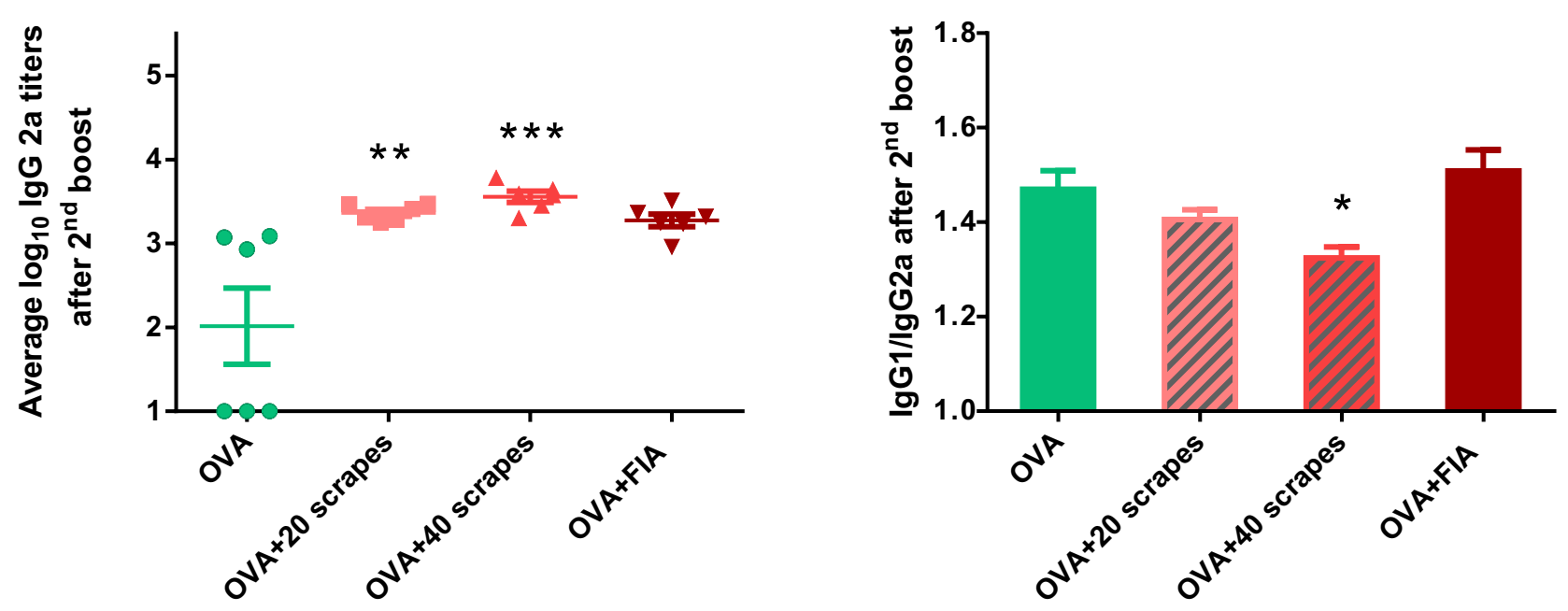